\title{
BAMBI inhibits inflammation through the activation of autophagy in experimental spinal cord injury
}

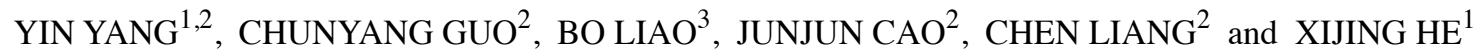 \\ ${ }^{1}$ Department of Orthopaedics, The Second Affiliated Hospital, Xi'an Jiaotong University, Xi'an, Shaanxi 710004; \\ ${ }^{2}$ The Second Department of Orthopedics, Xi'an Central Hospital, Xi'an, Shaanxi 710003; ${ }^{3}$ Department of Orthopaedics, \\ Tangdu Hospital, The Fourth Military Medical University, Xi'an, Shaanxi 710038, P.R. China
}

Received February 1, 2016; Accepted December 14, 2016

DOI: $10.3892 /$ ijmm.2016.2838

\begin{abstract}
Autophagy plays an important role in the progression of spinal cord injury (SCI). In this study, we aimed to examine the effects and potential mechanisms of action of BMP and activin membrane-bound inhibitor (BAMBI) in the progression of SCI. A rat model of SCI was established and the rats were injected with pLentiH1-BAMBI shRNA and pAd-BAMBI in the gray and white matter of the spinal cord at T8. After 14 days, motor function evaluation was measured according to the Basso Beattie Bresnahan (BBB) method and the number of motor neuron cell accounts in the anterior horns was measured by Nissl staining. The protein expression of levels light chain 3B (LC3B), Beclin 1, Bim and p62 were measured by western blot analysis. The concentrations of interleukin (IL)-1 $\beta$, IL-6 and IL-10 were measured by ELISA. The results revealed that BAMBI expression was significantly decreased in the rats with SCI. The BBB scores and the number of motor neuron cell accounts in the anterior horns were significantly decreased in the pLentiH1-BAMBI shRNA injection group, and were increased in the pAd-BAMBI injection group, suggesting a neuroprotective effect of BAMBI in SCI. Moreover, the increased expression levels of LC3B and Beclin 1, and the decreased expression of Bim and p62 indicated that autophagy was significantly induced in the pAd-BAMBI injection group. Moreover, the overexpression of BAMBI also decreased the expression of transforming growth factor- $\beta$ (TGF- $\beta$ ) and mammalian target of rapamycin (mTOR), and decreased the concentrations of IL-1 $\beta$, IL-6 and IL-10. These results indicate that BAMBI plays a neuroprotective role by decreasing inflammation and activating autophagy in rats with SCI.
\end{abstract}

Correspondence to: Dr Xijing He, Department of Orthopaedics, The Second Affiliated Hospital, Xi'an Jiaotong University, 157 Xiwu Road, Xi'an, Shaanxi 710004, P.R. China

E-mail: xijinghejt@163.com

Key words: BMP and activin membrane-bound inhibitor, spinal cord injury, autophagy, inflammation, transforming growth factor- $\beta$ / mechanistic target of rapamycin pathway

\section{Introduction}

Spinal cord injury (SCI) is a serious and debilitating disease that is characterized by axonal tissue degeneration and neurological dysfunction (1). SCI consists of two pathological phases, primary mechanical injury and secondary injury. Secondary injury consists of neuronal inflammation, demyelination, axonal degeneration and various degrees of oligodendrocyte and neuronal cell death, and plays an important role in the physical and functional deficits that occur after SCI (2). Therefore, a thorough elucidation of the mechanisms responsible for secondary injury is important in order to understand neurodegenerative disorders and to discover an appropriate therapeutic method.

Autophagy is an important cellular pathway characterized by the degradation of cytoplasmic proteins and organelles during development and under stress conditions (3). Autophagy plays a vital role in cellular homeostasis and has been shown to be involved in a number of biological processes and diseases, including SCI. For example, a previous study proved that vascular endothelial growth factor $\left(\mathrm{VEGF}_{165}\right)$ attenuated SCI by inhibiting inflammation and increasing autophagic activity (4). Another study reported that exendin-4 (Ex-4) significantly enhanced motor function in rats following SCI by promoting autophagy (5). In addition, pollen typhae has been shown to increase autophagic activity in damaged neural tissues following SCI (6).

Mammalian target of rapamycin (mTOR) is a serine/threonine protein kinase belonging to the phosphatidylinositol 3-kinase-related kinase protein (PIKK) family. mTOR is formed by two different protein complexes: mTORC1 and mTORC2 (7). mTOR1 regulates protein growth, autophagy and ribosomal biogenesis through integrating growth factor, whereas mTORC2 is involved in cell survival and cytoskeletal regulation (8). It has been proven that transforming growth factor- $\beta 1$ (TGF- $\beta 1$ ) inhibits autophagy by activating mTORC1 in fibroblasts (9). Consequently, TGF- $\beta /$ mTOR signaling may be a potential target for the treatment of SCI.

BMP and activin membrane-bound inhibitor (BAMBI) acts as a pseudo-receptor for the TGF- $\beta$ type I receptor family and as a negative modulator of TGF- $\beta$ kinase signaling due to its lack of the intracellular kinase domain (10). BAMBI elimination enhances alternative TGF- $\beta$ signaling in diabetic mice (10), whereas it has been reported that the expression of TGF- $\beta$ is 
markedly increased followng SCI (11). The inhibition of TGF- $\beta 1$ in injured neurons has been shown to enhance the growth of axons and to eventually promote functional recovery in rat models of SCI (12). However, whether BAMBI is associated with autophagy in the progression of SCI remains unknown.

Based on the above information, in the present study, we aimed to determine whether BAMBI alleviates motor function impairment following SCI and to explore the underlying mechanisms. The findings of our study may shed light into the molecular mechanisms responsible for the neuroprotective effects of BAMBI, and provide a potential approach for the treatment of SCI.

\section{Materials and methods}

Ethics statement. All animal procedures were approved by the Institutional Animal Care and Use Committee of the Second Affiliated Hospital, Xi'an Jiaotong University, Xi'an, China. Following the conclusion of the experiment, the mice were euthanized by an intraperitoneal injection of sodium pentobarbital $(50 \mathrm{mg} / \mathrm{kg})$.

Animals. Adult male Sprague-Dawley rats ( $\mathrm{n}=96 ; 250-300 \mathrm{~g})$ were obtained form Xi'an Jiaotong University Health Science Center (Xi'an, China). The rats were randomly divided into the following 4 groups (24 rats/group): The sham-operated (sham) group, the SCI group, the pLentiH1-BAMBI shRNA group (rats with SCI rats injected with pLentiH1-BAMBI shRNA vector), and the pAd-BAMBI group (rats with SCI were injected with pAd-BAMBI vector). Each group also contained 4 subgroups ( $n=6)$ for use in the following experiments: i) behavioral analysis of motor function; ii) western blot analysis and reverse transcription-quantitative PCR (RT-qPCR); iii) Nissl staining; and iv) enzyme-linked immunosorbent assay (ELISA).

Rat model of SCI.The rat model of SCI was developed according to the method of Hu et al (13). The rats were anesthetized with chloral hydrate $(300 \mathrm{mg} / \mathrm{kg})$. After the skin was sterilized with $75 \%$ alcohol, the lamina was exposed by cutting and separating the skin. A laminectomy was performed to expose the spinal cord at T9-T11. A spinal contusion was created using a $10 \mathrm{~g}$ weight impactor dropped from a height of $2 \mathrm{~cm}$. The skin was then closed in layers. The bladder of each rat was expressed 3 times a day until reflex bladder emptying was established. The sham-operated rats were subjected to the same process, but underwent the laminectomy only. The successful SCI model was verified as follows: flicking of the legs, spinal cord ischemia, flaccid paralysis of the lower limbs and the formation of tail sway reflex. All rats were housed individually in a room at $24^{\circ} \mathrm{C}$ under controlled light conditions.

Intraspinal microinjection. Lentiviral shRNA vectors, named pLentiH1-BAMBI shRNA, containing the BAMBI interference fragment were constructed. The recombinant adenoviral vector, named pAd-BAMBI, that overexpresses BAMBI was constructed and high viral titres were obtained. These two recombinant vectors were obtained from Genomeditech (Shanghai, China). Intraspinal microinjection was performed as previously described (14). The vectors, pLentiH1-BAMBI shRNA, pAd-BAMBI, and their corresponding control vectors were bilaterally injected into the gray and white matter of the spinal cord at T8 in the rats with SCI only once. Injections were made into the lateral funiculus $1.1 \mathrm{~mm}$ lateral to the midline at a depth of $1 \mathrm{~mm}$. For gray matter, bilateral injections were made at $0.5 \mathrm{~mm}$ lateral to the midline at a depth of $1.3 \mathrm{~mm}$ to target motor neurons in the ventral horn. Injections were made using a beveled glass micropipette (60 $\mu \mathrm{m}$ tip diameter) and nano-injector (Stoelting Co., Wood Dale, IL, USA). For each injection site, a volume of up to $1.0 \mu \mathrm{l}$ was slowly infused over a period of $4 \mathrm{~min}(100 \mathrm{nl} / \mathrm{min})$.

Behavioral analysis. The recovery of behavioral function was conducted using the Basso Beattie Bresnahan (BBB) locomotor rating scale at $1,3,7$ and 14 days after each treatment. The score was assessed by two independent observers. The score ranged from 0 to 21 . A score of 0 indicates complete hind limb paralysis and 21 indicates completely normal locomotion. The rats were placed in an open field. The 22-point scale included the monitoring of hind limb movements, trunk position and stability, stepping, co-ordination, paw placement, toe clearance and tail position.

Nissl staining. After the mice were anesthetized by an intraperitoneal injection of $10 \%$ chloral hydrate $(300 \mathrm{mg} / \mathrm{kg})$, the spinal cords from the T7-T10 level around the lesion epicenter were obtained and were incubated at $60^{\circ} \mathrm{C}$ for $30 \mathrm{~min}$. The spinal cord slices were then cleared with xylene 3 times and dehydrated with 100, 90 and $85 \%$ ethanol for 5 min each, and then with 80 and $70 \%$ ethanol for $3 \mathrm{~min}$ each. The slices were rinsed with distilled water for $1 \mathrm{~min}$, and stained with crystal violet dye (Sigma, St. Louis, MO, USA) in an oven at $37^{\circ} \mathrm{C}$ for $30 \mathrm{~min}$, rinsed with water for $8 \mathrm{~min}$, and rapidly separated with $95 \%$ ethanol. The sections were then incubated in anhydrous alcohol and xylene for $2 \times 5 \mathrm{~min}$. The slices were mounted with neutral gum.

$R T-q P C R$. The spinal cord injury tissues were collected following treatment and total RNA was extracted using TRIzol reagent (Invitrogen, Carlsbad, CA, USA). First-strand cDNA synthesis was carried out using the MMLV reverse transcriptase kit (Takara, Dalian, China). Quantitative (real-time) PCR (qPCR) was conducted using the SYBR Premix Ex Taq ${ }^{\mathrm{TM}}$ kit according to the manufacturer's instructions (Takara). All of the primers used in this study were synthesized by Sangon Biotech (Shanghai, China) and were as follows: BAMBI 5'-CCG TGC TGC TCA CCA AAG GTG-3', 5'-ATA CCT GTT TCC TTG TCC TGA-3'. Each individual sample was run in triplicate wells and the reactions were conducted in an ABI 7500 realtime PCR system Applied Biosystems, Carlsbad, CA, USA). The reaction conditions were an initial denaturation at $95^{\circ} \mathrm{C}$ for $30 \mathrm{sec}$ followed by 40 cycles at $95^{\circ} \mathrm{C}$ for $10 \mathrm{sec}$ and $60^{\circ} \mathrm{C}$ for $60 \mathrm{sec}$. The relative expression levels of the genes were calculated using the $2^{-\Delta \Delta C T}$ method. The 18S RNA gene was selected as a reference.

Western blot analysis. Spinal cords containing the injury site were collected following treatment and lysed with RIPA buffer containing PMSA (Sangon Biotech, Shanghai, China), and centrifuged at $12,000 \mathrm{x} \mathrm{g}$ for $20 \mathrm{~min}$ at $4^{\circ} \mathrm{C}$. The protein concentration was measured using a bicinchoninic acid (BCA) protein assay kit (Sangon Biotech). 
A

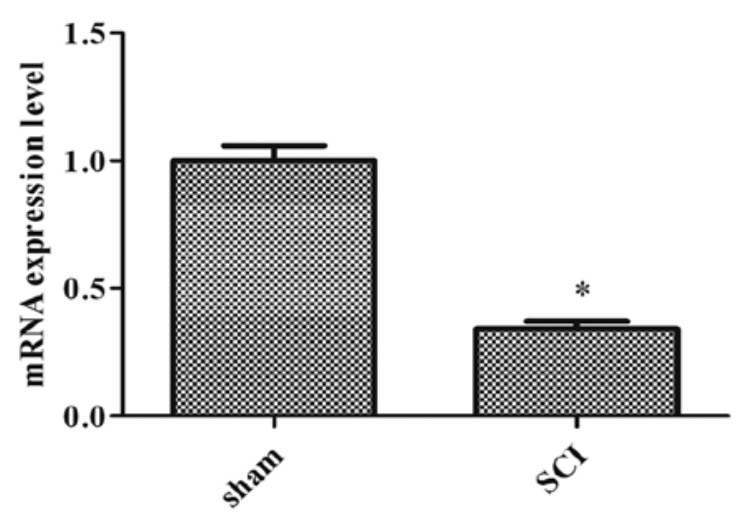

B

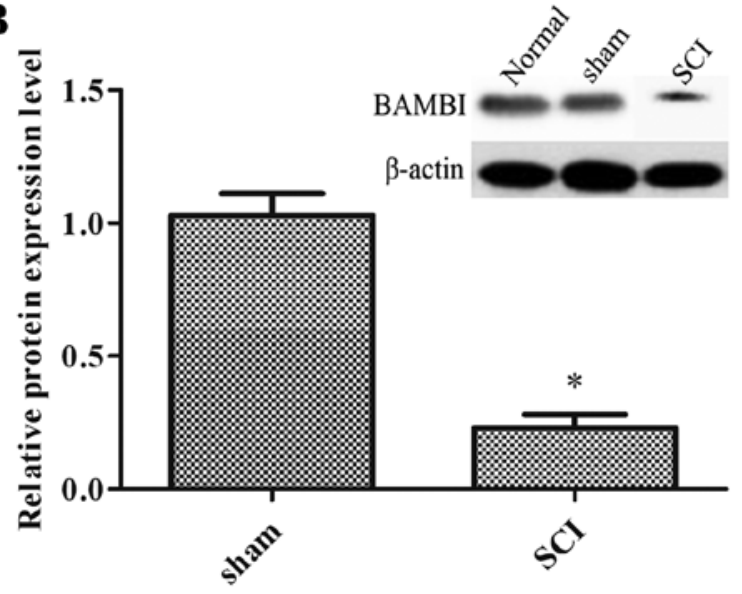

Figure 1. The expression of BMP and activin membrane-bound inhibitor (BAMBI) was decreased in rats with spinal cord injury (SCI). The spinal cords from the T7-T10 level around the lesion epicenter were obtained from rats with SCI. (A) The mRNA expression levels of BAMBI were measured by RT-qPCR. (B) The protein expression levels of BAMBI were measured by western blot analysis. ${ }^{*} \mathrm{P}<0.05$ vs. sham-operated (sham) group.

Total proteins $(30 \mu \mathrm{g})$ were separated by sodium dodecyl sulfate-polyacrylamide gel electrophoresis (SDS-PAGE) and transferred onto polyvinylidene difluoride (PVDF) membranes (Millipore, Darmstadt, Germany). The membranes were then probed with primary antibodies specific for rabbit polyclonal anti-rat BAMBI (Cat. no. ab203070; 1:200 dilution; Abcam, Cambridge, UK), rabbit monoclonal anti-rat Bim (Cat. no. ab7888; 1:1,000 dilution; Abcam), rabbit polyclonal anti-rat Beclin 1 (Cat. no. NB500-249; 1:1,000 dilution; Novus Biologicals, Littleton, CO, USA), rabbit polyclonal anti-rat levels light chain 3B (LC3B; Cat. no. a b48394; 1:1,000 dilution; Abcam), rabbit polyclonal anti-rat p62 (Cat. no. ab91526; 1:1,000 dilution; Abcam), rabbit polyclonal anti-rat TGF- $\beta$ (Cat. no. ab155264; 1:1,000 dilution; Abcam), rabbit polyclonal anti-rat mTOR (Cat. no. ab2833; 1:2,000 dilution; Abcam), rabbit polyclonal anti-rat p-p70s6k (Cat. no. ab2571, 1:250 dilution; Abcam). Following overnight incubation at $4^{\circ} \mathrm{C}$, the goat anti-rabbit IgG-HRP secondary antibody (Cat. no. ab6721; 1:2,000 dilution; Abcam) was added followed by incubation for $1 \mathrm{~h}$ at room temperature. The immunoreactive proteins were visualized using an ECL detection system (Amersham Biosciences, Amersham, UK).

ELISA. The spinal cords that included the injury site were collected and re-suspended in PBS. The tissues were then centrifuged at 3,000 rpm. The supernatant were collected and added to the appropriate wells for $2.5 \mathrm{~h}$ of incubation at room temperature. After washing with wash buffer 4 times, $100 \mu \mathrm{l}$ of $1 \mathrm{X}$ prepared biotinylated detection antibody were added to each well followed by $1 \mathrm{~h}$ of incubation. The prepared HRP-streptavidin solution (100 $\mu \mathrm{l})$ was added to each well followed by incubation for $45 \mathrm{~min}$. ELISA colorimetric TMB reagent $(100 \mu \mathrm{l})$ was added followed by incubation for $30 \mathrm{~min}$ in the dark with gentle shaking. Finally, stop solution (50 $\mu \mathrm{l})$ was added to each well and read at $450 \mathrm{~nm}$ immediately.

Statistical analysis. Statistical analysis was performed using the Student's unpaired t-test (SPSS release 19.0; SPSS, Inc., Chicago, IL, USA). Data are expressed as the means \pm SD.
A value of $\mathrm{P}<0.05$ was considered to indicate a statistically significant difference.

\section{Results}

The expression of BAMBI is decreased in rats with SCI. In order to confirm the role of BAMBI in SCI, a rat model of SCI was established. Compared with the sham-operated group, the mRNA and protein expression levels of BAMBI were significantly decreased in the spinal cord tissue of the rats with SCI $(\mathrm{P}<0.05)$ (Fig. 1).

Knockdown of BAMBI expression aggravates locomotor dysfunction and inhibits autophagy in rats with SCI. In order to examine the effects of BAMBI on SCI, BAMBI was knocked down by injecting the rats with SCI with a pLentiH1BAMBI shRNA injection. The interference efficiency of pLentiH1-BAMBI shRNA is shown in Fig. 2A. Compared with the sham-operated group, the expression of BAMBI was significantly decreased in the SCI group $(\mathrm{P}<0.05)$. When compared with the SCI group, the expression of BAMBI was decreased even further in the group that was injected with pLentiH1BAMBI shRNA $(\mathrm{P}<0.05)$. The results of the BBB score revealed that there was no locomotor dysfunction in the rats from the sham-operated group, but that locomotor function was significantly decreased in the rats with SCI (Fig. 2B). Even more severe hind limb locomotor dysfunction was observed in the rats that were injected with pLentiH1-BAMBI shRNA (Fig. 2B). As shown in Fig. 2C and D, compared with the sham-operated group, the anterior horn cells and the number of motor neurons were markedly decreased in the SCI group, particularly in the group infected with pLentiH1-BAMBI shRNA. Moreover, we also measured the expression levels of LC3B, Beclin 1, Bim and p62, which are related to the progression of autophagy. The results revealed that the expression levels of Bim and p62 were notably decreased, whereas those of LC3B and Beclin 1 were significantly increased in the SCI group $(\mathrm{P}<0.05)$. Compared with the SCI group, the expression levels of Bim and p62 were significantly increased, whereas those of LC3B and Beclin 1 

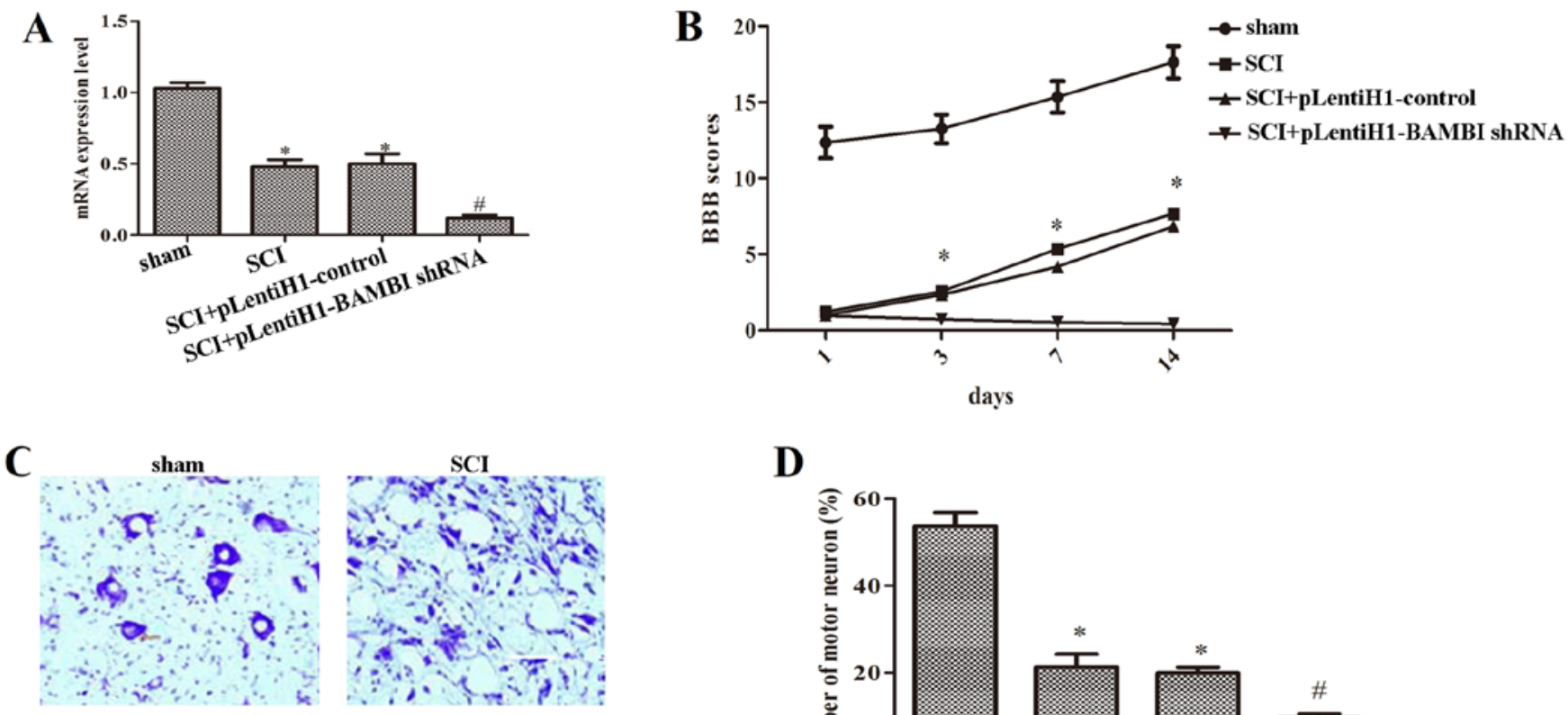

$\mathbf{D}$
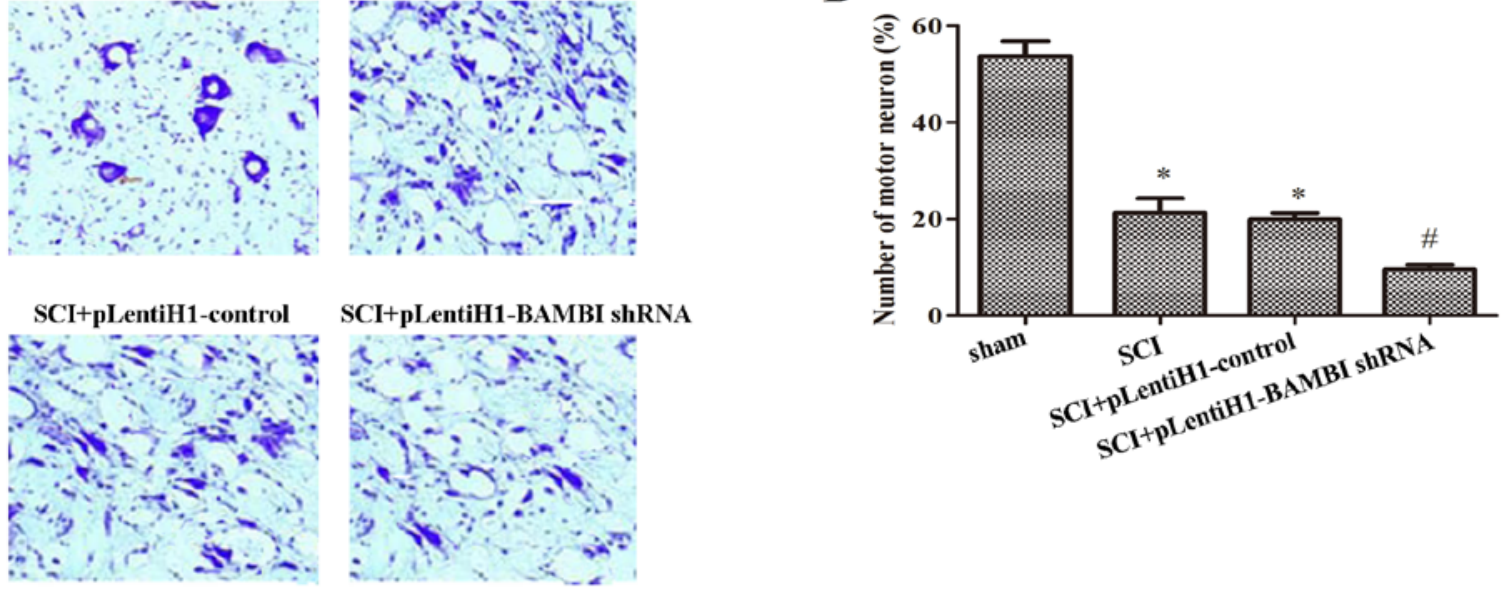

$\mathbf{E}$
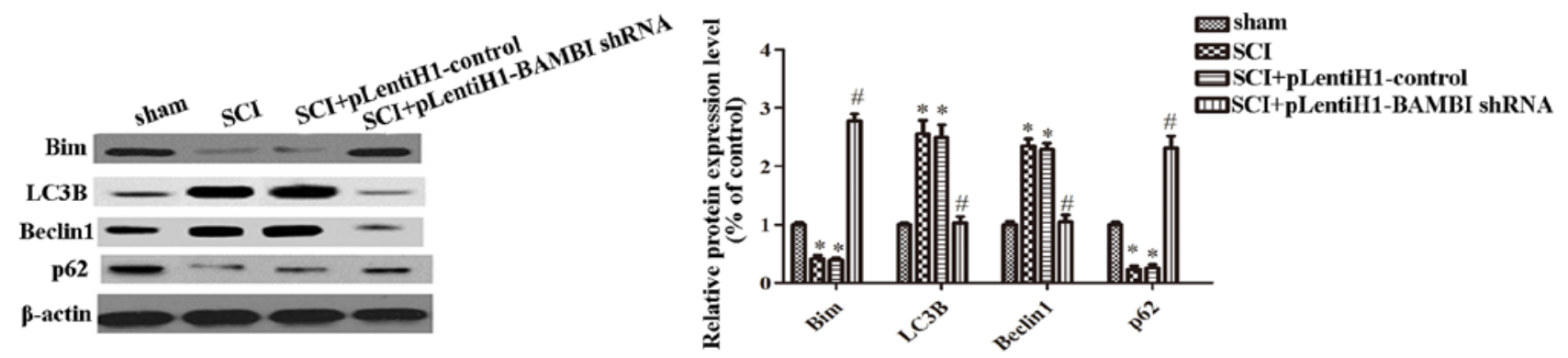

Figure 2. Silencing of BMP and activin membrane-bound inhibitor (BAMBI) expression aggravates locomotor dysfunction and inhibited autophagy in rats with spinal cord injury (SCI). Spinal cords containing the injury site were collected from rats with SCI injected with pLentH1-BAMBI for 14 days. (A) Interference efficiency of the pLentH1-BAMBI vector. (B) The recovery of behavioral function was assessed using the Basso Beattie Bresnahan (BBB) score on days 1, 3, 7 and 14. (C) Nissl staining of motor neurons in the large anterior horn. (D) Number of motor neuronal cell accounts in the anterior horns. (E) The protein expression of Bim, LC3B, Beclin 1 and p62 was measured by western blot analysis. ${ }^{*} \mathrm{P}<0.05$ vs. sham-operated (sham) group; ${ }^{\#} \mathrm{P}<0.05$ vs. SCI group.

were decreased in the group that was injected with pLentiH1 BAMBI shRNA (Fig. 2E). From these results, it can be concluded that the silencing of BAMBI expression leads to the aggravation of locomotor dysfunction and the inhibition of autophagy.

The overexpression of BAMBI attenuates locomotor dysfunction and increases autophagy in rats with SCI.To further analyze the effects of BAMBI on SCI, BAMBI was overexpressed by injecting the rats with SCI with a BAMBI overexpression vector (pAd-BAMBI). The overexpression efficiency of pAdBAMBI is shown in Fig. 3A. Compared with the SCI group, the expression of BAMBI was significantly increased in the group injected with pAd-BAMBI (Fig. 3A). As shown in Fig. 3B, we found that when the rats were injected with pAd-BAMBI, the BBB score was significantly increased on days 7 and 14 following the induction of SCI. As shown in Fig. 3C and D, the number of motor neurons in the anterior horn cells were markedly increased when the rats were injected with pAdBAMBI. These results suggested that the overexpression of BAMBI attenuated motor dysfunction caused by SCI. We also measured the expression of proteins related to autophagy. The expression levels of Bim and p62 were significantly decreased, whereas those of LC3B and Beclin 1 were notably increased in the group injected with pAd-BAMBI $(\mathrm{P}<0.05)$ (Fig. 3E). The above-mentioned results indicate that BAMBI plays a positive role in SCI.

Overexpression of BAMBI inhibits inflammation and the activation of the MTOR signaling pathway in rats with SCI. To further elucidate the underlying mechanisms responsible for the protective effects of BAMBI in rats with SCI, we analyzed the levels of cytokines, including IL-1 $\beta$, IL-6, IL-10, and the protein expres- 
$\mathbf{A}$

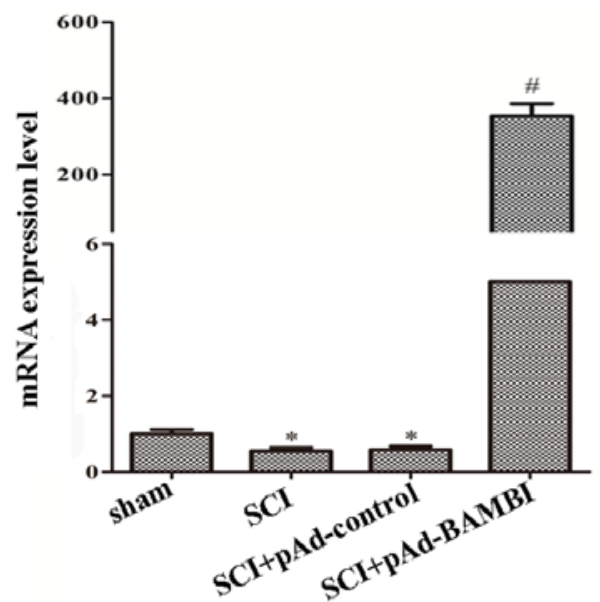

C
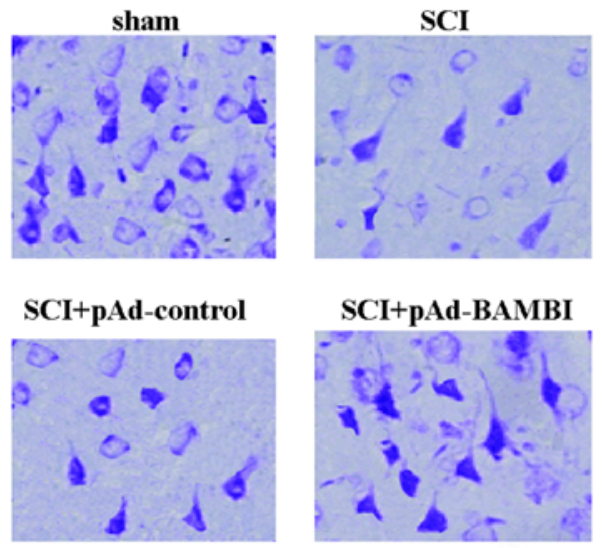

B

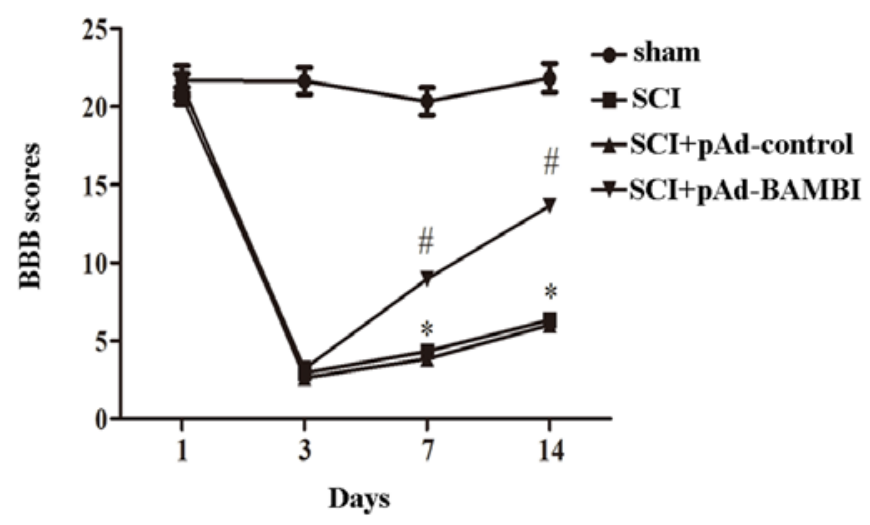

D
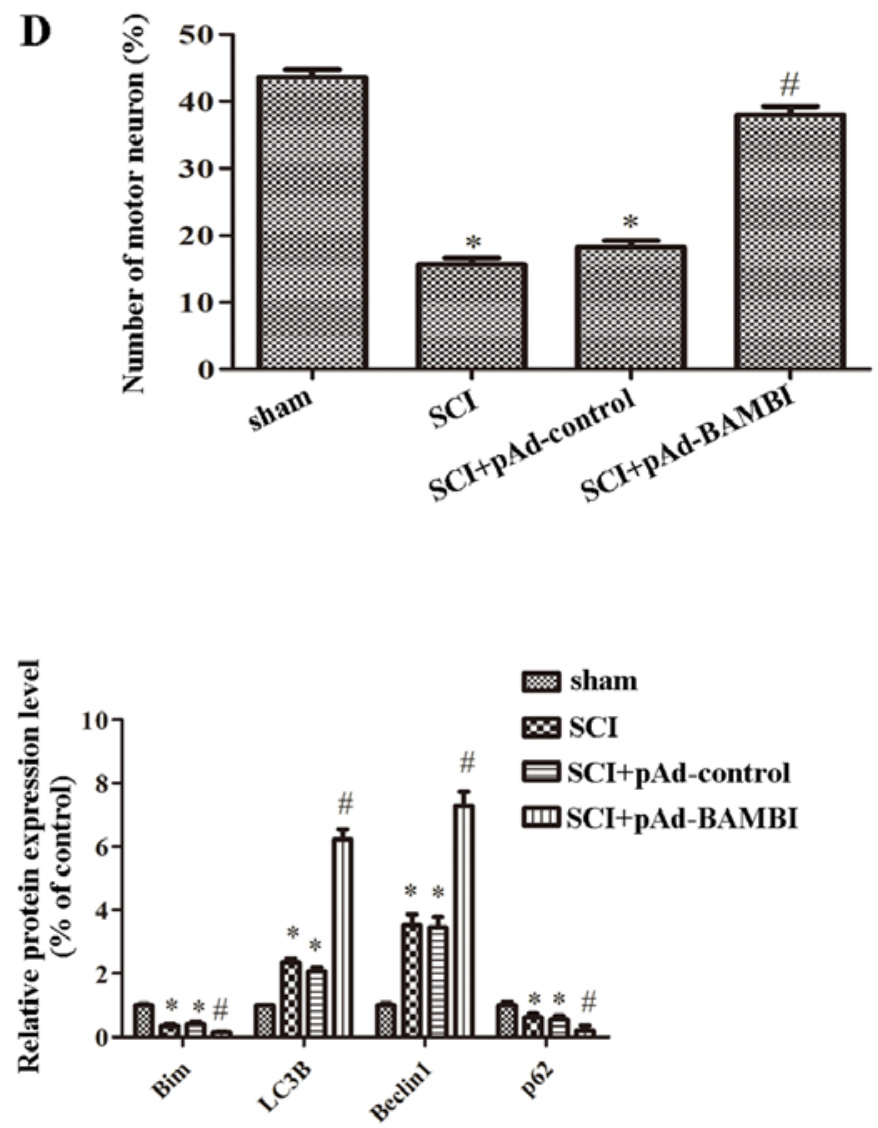

Figure 3. Overexpression of BMP and activin membrane-bound inhibitor (BAMBI) attenuates locomotor dysfunction and increases autophagy in rats with spinal cord injury (SCI). Spinal cords containing the injury site were collected from rats with SCI injected with pAd-BAMBI for 14 days. (A) Overexpression efficiency of the pAd-BAMBI vector. (B) The recovery of behavioral function was assessed using the Basso Beattie Bresnahan (BBB) score on days 1,3,7 and 14. (C) Nissl staining of motor neurons in the large anterior horn. (D) Number of motor neuronal cell accounts in the anterior horns. (E) The protein expression of Bim, LC3B, Beclin 1 and p62 was measured by western blot analysis. "P<0.05 vs. sham-operated group; ${ }^{*} \mathrm{P}<0.05$ vs. SCI group.

sion levels in the mTOR signaling pathway, which is related to autophagy. When compared with the SCI group, the concentrations of IL-1 $\beta$, IL- 6 and IL-10 were significantly decreased in the group injected with pAd-BAMBI $(\mathrm{P}<0.05)$ (Fig. 4A). As shown in Fig. 4B, the expression levels of TGF- $\beta$, mTOR and p-p70s6k were markedly decreased in the group injected with pAd-BAMBI $(\mathrm{P}<0.05)$. These results indicate that the overexpression of BAMBI inhibits inflammation and the activation of the mTOR signaling pathway in rats with SCI.

\section{Discussion}

SCI is regarded as a major health concern and is a frequent cause of disability and mortality worldwide (15). A number of studies have proven that neuroprotection and neurorecovery play an important role in the treatment of SCI. For example, apigenin may be a potential agent for the treatment of SCI by promoting the recovery of rat neuronal function (16). Also, in another study, the inhibition of miR-20a expression was 

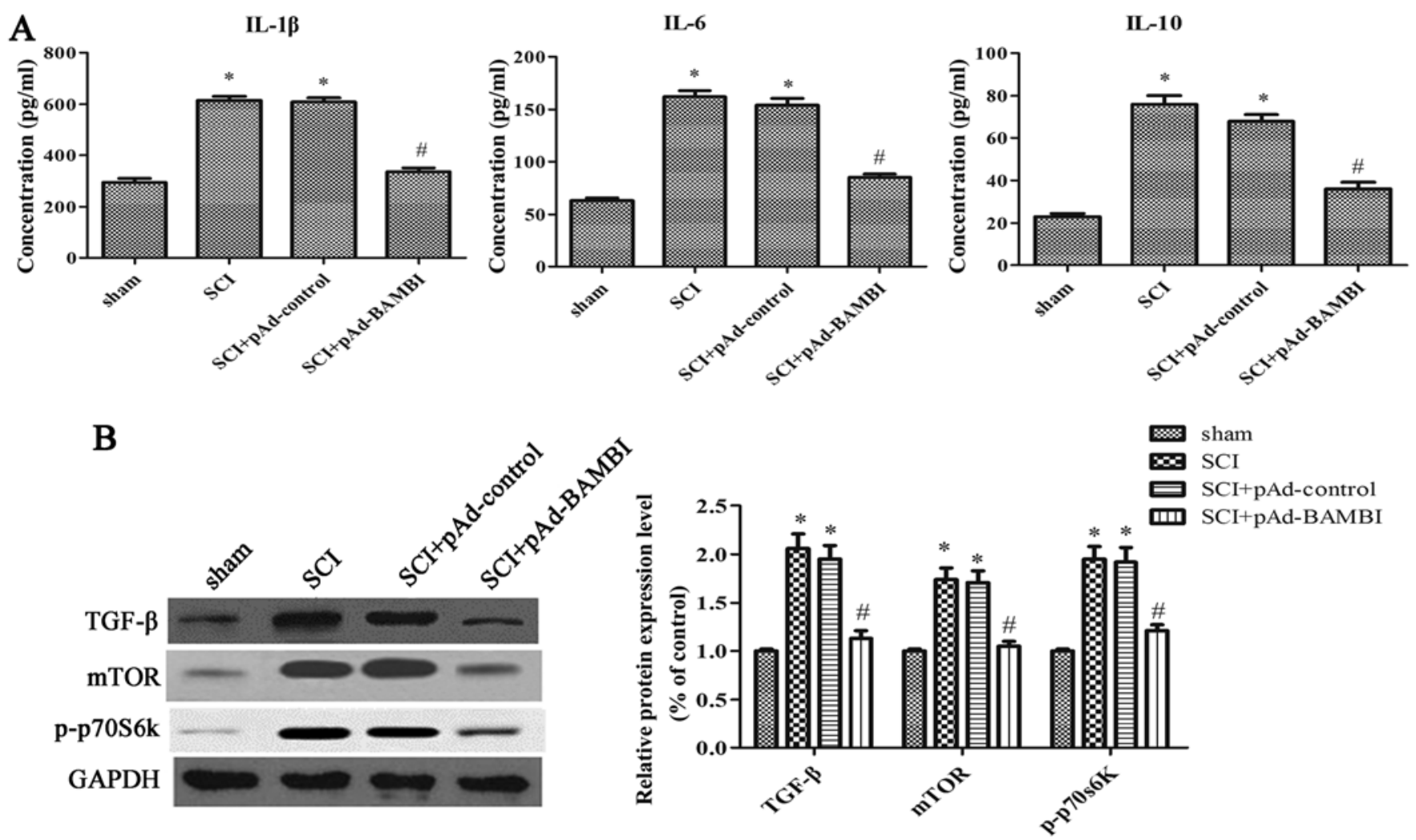

Figure 4. Overexpression of BMP and activin membrane-bound inhibitor (BAMBI) inhibits inflammation and the mammalian target of rapamycin (mTOR) signaling pathway in rats with spinal cord injury (SCI). Spinal cords containing the injury site were collected from rats with SCI injected with pAd-BAMBI for 14 days. (A) The contents of interleukin (IL)-1 $\beta$ ), IL-6 and IL-10 were measured by ELISA. (B) The expression levels of transforming growth factor- $\beta$ (TGF- $\beta$ ), mTOR and p-p70S6k were measured by western blot analysis. ${ }^{*} \mathrm{P}<0.05$ vs. sham-operated (sham) group; ${ }^{*} \mathrm{P}<0.05$ vs. SCI group.

shown to induce definitive motor neuron survival and neurogenesis, and animals with SCI exhibited improved functional deficit (17). In the present study, our results confirmed that the overexpression of BAMBI attenuated motor dysfunction and decreased inflammation and induced autophagy in rats with SCI.

An increasing number of studies have verified that BAMBI plays a vital role in various diseases. BAMBI acts as a negative modulator of myocardial remodeling under pressure overload (18). The overexpression of BAMBI has been shown to inhibit keloid growth through the suppression of TGF- $\beta 1$-induced fibroblast cell proliferation and the excessive accumulation of collagen I (19). This study demonstrated that BAMBI was downregulated in rats with SCI, and the overexpression of BAMBI promoted functional neurobehavioral recovery, which was evidenced by the increased BBB score and the number of motor neurons in rats with SCI.

Autophagy has been shown to exert a neuroprotective effect in rats with acute SCI (20). It has been demonstrated that LC3B and Beclin 1 are two reliable markers for the progression of autophagy (21). For example, the expression of Beclin 1 has been shown to be markedly increased in damaged neural tissue and this induces autophagic cell death following SCI (22). Treatment with rammycin has been shown to induce autophagy by increasing the expression levels of LC3 and Beclin 1 following SCI (23). Bim and p62 are also two major factors in autophagy. Bim inhibits autophagy by recruiting Beclin 1 to microtubules (24). The loss of total Bim in IL-7-deprived
$\mathrm{T}$ cells has beeb shown to cause a delayed degradative phase of autophagy (25). p62 acts as a cellular metabolic switch in autophagy (26) and exerts a protective effect against polyQ-induced neurodegeneration through the autophagic degradation of polyQ protein oligomers (27). Our results also indicated that the overexpression of BAMBI significantly increased the expression levels of LC3B and Beclin 1, and decreased the expression of Bim and p62 in rats with SCI. This result indicated that the overexpression of BAMBI protected the rats from SCI by activating autophagy.

A growing number of studies have demonstrated that mTOR signaling is involved in autophagy (7). For example, TNF alpha induced protein 3 (TNFAIP3) inhibits mTOR signaling and promotes autophagy (28). Another study reported that Che-1 induced autophagy by inhibiting the mTOR pathway (29). mTOR has been proven to promote compensatory neuronal sprouting important for recovery following nerve injury (30). An increased Rheb expression has been shown to contribute to mTOR activation in SCI (31). Our results also revealed that the overexpression of BAMBI inhibited the expression of TGF- $\beta$, thus leading to the inhibition of mTOR signaling in rats with SCI. Furthermore, we also found that the overexpression of BAMBI decreased inflammation in rats with SCI. A previous study also demonstrated that TGF- $\beta$ activated the NF- $\kappa B$ pathway to promote osteoclast survival (32). TGF- $\beta$ is regarded as a pro-inflammatory agent by recruiting and activating resting monocytes (12). The inhibition of TGF- $\beta 1$ enhances the activation of macrophages in rat models of SCI (12). Therefore, we 
concluded that the overexpression of BAMBI causes the downregulation of TGF- $\beta$, leading to the inhibition of the NF- $\kappa \mathrm{B}$ pathway, further resulting in the decrease in the levels of IL-1 $\beta$, IL-6 and IL-10. Taken together, our results demonstrate that the overexpression of BAMBI decreases inflammation and induces autophagy by inhibiting mTOR signaling in rats with SCI.

In conclusion, the present study suggests that the overexpression of BAMBI exerts a neuroprotective and neurorecovery effect on SCI. Furthermore, the upregulation of BAMBI decreases inflammation and induces autophagy by inhibiting mTOR signaling in rats with SCI. Our findings reveal the molecular mechanisms responsible for the neuroprotective roles of BAMBI, and may provide a potential therapy for SCI.

\section{References}

1. Chen B, He J, Yang H, Zhang Q, Zhang L, Zhang X, Xie E, Liu C, Zhang R, Wang Y, et al: Repair of spinal cord injury by implantation of bFGF-incorporated HEMA-MOETACL hydrogel in rats. Sci Rep 5: 9017, 2015.

2. Su M, Guan H, Zhang F, Gao Y, Teng X and Yang W: HDAC6 regulates the chaperone-mediated autophagy to prevent oxidative damage in injured neurons after experimental spinal cord injury. Oxid Med Cell Longev 2016: 7263736, 2016.

3. Siracusa R, Paterniti I, Bruschetta G, Cordaro M, Impellizzeri D, Crupi R, Cuzzocrea S and Esposito E: The association of palmitoylethanolamide with luteolin decreases autophagy in spinal cord injury. Mol Neurobiol 53: 3783-3792, 2016.

4. Wang H, Wang Y, Li D, Liu Z, Zhao Z, Han D, Yuan Y, Bi J and Mei X: VEGF inhibits the inflammation in spinal cord injury through activation of autophagy. Biochem Biophys Res Commun 464: 453-458, 2015.

5. Li HT, Zhao XZ, Zhang XR, Li G, Jia ZQ, Sun P, Wang JQ, Fan ZK and Lv G: Exendin-4 enhances motor function recovery via promotion of autophagy and inhibition of neuronal apoptosis after spinal cord injury in rats. Mol Neurobiol 53: 4073-4082, 2016

6. Wang W, Guo Z, Xu Z, Meng Q, Chen C, Zhang Y and Cao X: Effect of pollen typhae on inhibiting autophagy in spinal cord injury of rats and its mechanisms. Int J Clin Exp Pathol 8: 2375-2383, 2015.

7. Perluigi M, Di Domenico F and Butterfield DA: mTOR signaling in aging and neurodegeneration: At the crossroad between metabolism dysfunction and impairment of autophagy. Neurobiol Dis 84: 39-49, 2015

8. Din FV, Valanciute A, Houde VP, Zibrova D, Green KA, Sakamoto K, Alessi DR and Dunlop MG: Aspirin inhibits mTOR signaling, activates AMP-activated protein kinase, and induces autophagy in colorectal cancer cells. Gastroenterology 142: 1504-1515.e1503, 2012.

9. Patel AS, Lin L, Geyer A, Haspel JA, An CH, Cao J, Rosas IO and Morse D: Autophagy in idiopathic pulmonary fibrosis. PLoS One 7: e41394, 2012.

10. Fan Y, Li X, Xiao W, Fu J, Harris RC, Lindenmeyer M, Cohen CD, Guillot N, Baron MH, Wang N, et al: BAMBI elimination enhances alternative TGF- $\beta$ signaling and glomerular dysfunction in diabetic mice. Diabetes 64: 2220-2233, 2015.

11. Hellal F, Hurtado A, Ruschel J, Flynn KC, Laskowski CJ, Umlauf M, Kapitein LC, Strikis D, Lemmon V, Bixby J, et al: Microtubule stabilization reduces scarring and causes axon regeneration after spinal cord injury. Science 331: 928-931, 2011

12. Kohta M, Kohmura E and Yamashita T: Inhibition of TGF-beta1 promotes function recovery after spinal cord injury. Neurosci Res 65: 393-401, 2009.

13. Hu JZ, Long H, Wu T-D, Zhou Y and Lu H-B: The effect of estrogen-related receptor $\alpha$ on the regulation of angiogenesis after spinal cord injury. Neuroscience 290: 570-580, 2015.
14. Yu CG, Yezierski RP, Joshi A, Raza K, Li Y and Geddes JW: Involvement of ERK2 in traumatic spinal cord injury. J Neurochem 113: 131-142, 2010.

15. Li L, Jiang HK, Li YP and Guo YP: Hydrogen sulfide protects spinal cord and induces autophagy via $\mathrm{miR}-30 \mathrm{c}$ in a rat model of spinal cord ischemia-reperfusion injury. J Biomed Sci 22: 50 , 2015.

16. Zhang F, Li F and Chen G: Neuroprotective effect of apigenin in rats after contusive sipinal cord injury. Neurol Sci 35: 583-588, 2014.

17. Jee MK, Jung JS, Im YB, Jung SJ and Kang SK: Silencing of miR-20a is crucial for Ngn1-mediated neuroprotection in injured spinal cord. Hum Gene Ther 23: 508-520, 2012.

18. Villar AV, García R, Llano M, Cobo M, Merino D, Lantero A, Tramullas M, Hurlé JM, Hurlé MA and Nistal JF: BAMBI (BMP and activin membrane-bound inhibitor) protects the murine heart from pressure-overload biomechanical stress by restraining TGF- $\beta$ signaling. Biochim Biophys Acta 1832: 323-335, 2013.

19. Lin L, Wang Y, Liu W and Huang Y: BAMBI inhibits skin fibrosis in keloid through suppressing TGF- $\beta 1$-induced hypernomic fibroblast cell proliferation and excessive accumulation of collagen I. Int J Clin Exp Med 8: 13227-13234, 2015.

20. Tang P, Hou H, Zhang L, Lan X, Mao Z, Liu D, He C, Du H and Zhang L: Autophagy reduces neuronal damage and promotes locomotor recovery via inhibition of apoptosis after spinal cord injury in rats. Mol Neurobiol 49: 276-287, 2014.

21. Barth S, Glick D and Macleod KF: Autophagy: Assays and artifacts. J Pathol 221: 117-124, 2010.

22. Kanno H, Ozawa H, Sekiguchi A and Itoi E: Spinal cord injury induces upregulation of Beclin 1 and promotes autophagic cell death. Neurobiol Dis 33: 143-148, 2009.

23. Sekiguchi A, Kanno H, Ozawa H, Yamaya S and Itoi E: Rapamycin promotes autophagy and reduces neural tissue damage and locomotor impairment after spinal cord injury in mice. J Neurotrauma 29: 946-956, 2012

24. Luo S, Garcia-Arencibia M, Zhao R, Puri C, Toh PP, Sadiq O and Rubinsztein DC: Bim inhibits autophagy by recruiting Beclin 1 to microtubules. Mol Cell 47: 359-370, 2012.

25. Ruppert SM, Li W, Zhang G, Carlson AL, Limaye A, Durum SK and Khaled AR: The major isoforms of Bim contribute to distinct biological activities that govern the processes of autophagy and apoptosis in interleukin-7 dependent lymphocytes. Biochim Biophys Acta 1823: 1877-1893, 2012.

26. Moscat J and Diaz-Meco MT: Feedback on fat: p62-mTORC1autophagy connections. Cell 147: 724-727, 2011.

27. Saitoh Y, Fujikake N, Okamoto Y, Popiel HA, Hatanaka Y, Ueyama M, Suzuki M, Gaumer S, Murata M, Wada K and Nagai Y: p62 plays a protective role in the autophagic degradation of polyglutamine protein oligomers in polyglutamine disease model flies. J Biol Chem 290: 1442-1453, 2015.

28. Matsuzawa Y, Oshima S, Takahara M, Maeyashiki C, Nemoto Y, Kobayashi M, Nibe Y, Nozaki K, Nagaishi T, Okamoto R, et al: TNFAIP3 promotes survival of CD4 T cells by restricting MTOR and promoting autophagy. Autophagy 11: 1052-1062, 2015.

29. Desantis A, Bruno T, Catena V, De Nicola F, Goeman F, Iezzi S, Sorino C, Ponzoni M, Bossi G, Federico V, et al: Che-1-induced inhibition of mTOR pathway enables stress-induced autophagy. EMBO J 34: 1214-1230, 2015.

30. Park KK, Liu K, Hu Y, Smith PD, Wang C, Cai B, Xu B, Connolly L, Kramvis I, Sahin M and He Z: Promoting axon regeneration in the adult CNS by modulation of the PTEN/mTOR pathway. Science 322: 963-966, 2008.

31. Codeluppi S, Svensson CI, Hefferan MP, Valencia F, Silldorff MD, Oshiro M, Marsala M and Pasquale EB: The Rheb-mTOR pathway is upregulated in reactive astrocytes of the injured spinal cord. J Neurosci 29: 1093-1104, 2009.

32. Gingery A, Bradley EW, Pederson L, Ruan M, Horwood NJ and Oursler MJ: TGF- $\beta$ coordinately activates TAK1/MEK/AKT/ NFKB and SMAD pathways to promote osteoclast survival. Exp Cell Res 314: 2725-2738, 2008 